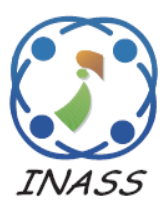

\title{
Wind Energy Output Prediction Model Based on DPSO-BP Neural Network
}

\author{
Dhari Y. Mahmood ${ }^{1}$ \\ Mohammed Kdair Abd ${ }^{1 *}$ \\ Kanaan A. Jalal ${ }^{1}$ \\ ${ }^{1}$ Department of Electrical Engineering, University of Technology - Iraq, Baghdad, Iraq \\ * Corresponding author's Email: 30098@uotechnology.edu.iq
}

\begin{abstract}
Wind Energy Output Prediction (WEOP) has an important impact on the integration of wind energy systems in the power grid, the management, power systems dispatching, safe and stable operation. Short-term probabilistic WEOP is a perfect choice to increase the stability of the power grid. Still, it has a high error because of uncertainty factors such as wind speed, and very important to find out a method to increase the fineness of predicting. Therefore, in this paper, the main contribution is to design an intelligent model capable of wind energy generation prediction. This aim is achieved by adopting a new Developed Particle Swarm optimization algorithm based on proposes a new inertia weight called DPSO and Back-Propagation Neural Network (DPSO-BPNN). The proposed models were compared and verified through MATLAB software test to prove the superiority over other models. The obtained results of the proposed model show a fast convergence and high prediction accuracy. Also, the error rate was improved compared with the published research by up to $98 \%$.
\end{abstract}

Keywords: Wind energy, Output prediction, Artificial neural network, Fuzzy logic, PSO.

\section{Introduction}

Wind farms cover a large proportion of the loads that depend on renewable energy sources due to its more worldwide and availability of winds permanently and throughout the year. The exact prediction of wind energy leads to enhance efficiency and reliability of the power supplies from wind farms. It can provide a reference for dispatching management of electrical power networks and arrange power supply start-up methods prior. Also, putting the emergency plans to ensure the stable and safe operation of the electrical power network. Wind energy has randomness and fluctuation, and the increase of wind power penetration rate brings severe challenges to the safety, stability, reliable operation, and power quality of the power grid [1].

Improving wind energy forecasting accuracy is conducive to the online bidding of wind energy, raising the upper limit of wind energy penetration thresholds and reducing wind energy integration's adverse impact on the power system. Also, the conducive to the long-term development of wind energy.
Wind power systems have developed rapidly, but because of the fluctuation, randomness, and intermittent nature of wind power, wind power has instability. The wind power prediction methods can be divided into physics, statistics, and learning methods [2]. The physical method generally predicts the wind power based on the Numerical Weather Forecast (NWF) data of the wind farm. This method requires detailed physical information and related meteorological data around the wind farm, and less historical data is needed for modeling. However, the NWP always suffers from onerousness in gaining data and its limited-spatial accuracy [3]. In reference [4], the statistical methods include the Kalman filtering method, which combines observations and model prediction recursively to minimize the corresponding-biases.

Furthermore, the percentage error between simulated and measured wind energy values showed relatively low and led a very appreciable development. Grey prediction method in reference [5] for ultra-short-term wind power prediction is presented, but the computational burden should be kept low to enable fast operational decisions. Where 
was not achieved due to the increased error rate. The learning methods mainly include spatial correlation method, time series models and soft computing techniques, support vector machine method, neural network method, extreme learning machine method [6-10], etc.

The above methods have their characteristics, but the same prediction method may not have good prediction performance in all wind farm operating scenarios, and there are specific application limitations. Combined prediction can make full use of the information of different single prediction methods, improve the prediction accuracy of wind energy and improve the applicability of prediction models [11]. So, it has received widespread attention. Combination prediction methods are divided into linear combination methods and non-linear combination methods. The core of the linear combination method is to solve the weight values of all the individual prediction methods.

Generally, research shows that most literature determines weight coefficients through some optimization criterion. In [12] calculates weight coefficients based on the squared error and the minimum criterion. Therefore, constant fluctuation and change in wind speed and air density cause a difference in the energy produced by wind farms. In [13], a neural network with a two-hidden layer has been proposed, which can be represented as a useful tool to predict the wind energy output, which improves various prediction indicators. The obtained results show that the estimated wind energy values with the proposed network are in rather agree with the experimentally measured values.

Reference [14] presented an approach of a nonlinear combination of wind energy prediction based on a multi-output support vector machine and grey wolf optimizer (GWO-MSVM) model to improve prediction terms of multiple-error metrics, including fractional bias and direction accuracy. In [15] first decomposed the wind speed by Empirical Mode Decomposition (EMD) and used various adaptive methods to predict the low-frequency components and combination of Artificial Neural Network (ANN) model to perform combined prediction for the highfrequency components. The final predicted assessment was equal to the algebraic summations of all high-low frequency components, and the results indicate that the accuracy of the prediction model is low.

In [16], the Gravitational Search Algorithm (GSA) was used to optimize the parameters of the Least Squares Support Vector Machine model (LSSVM) and Ensemble Empirical Mode Decomposition - Permutation Entropy (EEMD-PE) for increased prediction accuracy of wind power generation. If the wind power prediction error is large, it will affect the safe and stable operation and power system dispatching. Therefore, improving the prediction accuracy of wind energy has become a primary task.

Nature intermittent and random wind power, make it difficult to achieve accurate prediction using a single prediction model. In reference [17], a hybrid model (IDA-SVM) combining Improved Dragonfly Algorithm (IDA) and Support Vector Machin (SVM) is established to predict short-term wind power. The proposed model has shown better prediction performance compared with the other models such as SVM optimized by DA (DA-SVM), SVM optimized by Genetic Algorithm (GA-SVM), SVM optimized by Grid search method (Grid-SVM), BPNN, and Gaussian Process Regression (GPR). However, the accuracy of the proposed model may decrease with large-samples.

Reference [18] presented a hybrid approach combining the Autoregressive Integrated Moving Average (ARIMA) model and the Radial Basis Function Neural Network (RBFNN) model in addition to BPNN models to increase the predicting accuracy of wind power, as well as solution convergence. The results of the case study show that the proposed models are suitable for short-term predicting applications. However, conclusions show that the performance of the NN model in wind energy predicting may be deceptive. A thorough investigation is needed on the selection of NN types and parameter models. Therefore, it is necessary to carry out a hybrid between the $\mathrm{NN}$ and other intelligent methods.

In this paper, the BPNN algorithm based on the new development of PSO is used in wind energy prediction. It combines local optimization characteristics of the BPNN and global optimization of the PSO algorithm. Therefore, this paper proposed three models of intelligent algorithms: Fuzzy Neural Network (FNN), DPSO-FNN, and DPSO-BPNN, that can deliver wind predicting for a time horizon of short-time.

However, improved predicting of wind energy can be regarded as one of the most accurate and efficient methods to solve different problems. It includes the effective market of design and electricity clearing, power quality, costs, standards interconnection, transmission capacity, real-time system operations, service requirements, stability, and power system reliability.

In this paper, the features are listed as follows: 
a) Design of a new intelligent model capable of wind energy generation prediction for 48 hours and 72 hours.

b) New development on PSO algorithm by modifying the initial weights (Wnew) based on linearly decreasing and adopting the BPNN to get the optimum solution of WEOP and provide more realistic analyses.

c) DPSO will be employed to improve the performance of the BPNN and make it effective where quickly reduce learning and generalization errors.

d) The proposed method will be thoroughly tested and benchmarked on real wind energy data with reference [17, 18].

e) Study the optimization results obtained from the proposed models, including a formulation of the WEOP problem, and compare it with error rates.

f) Use criteria of prediction accuracy to evaluate the performance of the proposed model. The criteria of prediction accuracy, in this article, will include the Mean Absolute-Error (MAE), Root Mean Square-Error (RMSE), and Mean Absolute Percentage-Error (MAPE).

g) The results of prediction solutions for wind energy output obtained from DPSO-BPNN will be compared with previously published research papers.

This article will be organized as follows; Section two presented the related works. In section three introduced a new method for the development of PSO depended on inertia weight. Section four presents the proposed models and implementation steps of the proposed approaches. In Section five, we presented an analysis for the results and prediction performance of the proposed models, including a study of two cases, and compared the results with previously published methods with plotting the results. Lastly, conclusions and future work will be given in Section six.

\section{Related works}

Wind Energy has characteristics of haphazardness variable and uncontrollability. WEOP can be an important part and challenge of electrical energy system construction in the coming years. The WEOP method is important in identifying and power systems operation with an extensive penetration of wind energy.

WEOP can be classified according to; Time horizons, methods and principles, predict object, input data, and evaluation criteria about the inherent uncertainty for WEOP. As the wind farms are increased, the aggregated WEOP methods became faster for a region with several wind farms distributed [19]. The prediction system can be divided into three categories: spot prediction, wind farm prediction, or regional prediction [20]. The wind position can be enhanced by using prediction tools that can deal with the intermittent of the wind nature.

Wind energy can be scheduled using accurate wind prediction as the cost effects of wind can be greatly reduced. Thus, improving the performance of wind energy and prediction tools has a major economic impact on the electric power grid by increasing the penetration of wind energy into the grid. Many authors have devoted their efforts to improving wind energy predicting techniques, and several of the wind farm predicting models have been developed in various locations around the world [21]. Besides, accurate numerical weather forecasting has been proposed for short-term wind predicting [22-24]. In reference [25], the described system has a modular framework that provides wind forecasting for a time horizon of up to 24 hours.

Many kinds of literature and studies focused on long-term forecasting, where reference [26] proposed a long-term wind power forecasting model based on multi meteorological variables with data compensation. The RBF neural network model is built between meteorological variables and the power to get annuals of suitable power data. The problem of long-term wind speed and power forecasting based on meteorological information is proposed in [27]. It was solved using three types of recurrent neural networks as consideration predictive models.

Furthermore, in [28] are suggested recurrent forecast models outperform the atmospheric and time-series models to reduce computational complexity and storage requirements. In reference [29], a prediction model of wind farm power generators is presented based on Fuzzy modeling. A short-term WEOP model is proposed and derived from raw data of wind farms. This model preserves good prediction accuracy and provides an interpretable model that contains several roles from which it may reveal a useful qualitative description of the prediction system.

Reference [30] used aero structural simulations of generic 1.5 MW turbines to rank the atmospheric effect on the output power. These data are used to train regression that forecasts the turbine response for any combination of wind speed. In reference [31] presented an approach based on weather data and analysis of the main parameters with their correlation to energy output. They used a generic programming tool modeler based on the symbolic regression to deal with the different parameters. 


\section{Development of particle swarm optimization (DPSO)}

The proposed PSO algorithm is a random optimization technique that depends on swarm intelligent mobility $[32,33]$. In this technique, the collection of random particles is initialized, and then the search for the optimal solution is done by iteration.

In each iteration, the velocity and position of the particle are continuously updated by tracking the solution of individual optimal value $\left(g_{\text {best }}\right)$ and the global optimal solution value $\left(p_{\text {best }}\right)$.

Each particle has a vector of velocity $(V)$ and vector of position $(X)$, which are updated by the equations as shown below:

$$
\begin{gathered}
\begin{array}{r}
V_{n, m}^{(i+1)}=w \cdot V_{n, m}^{i}+c_{1} \cdot r_{1}\left(g_{\text {best }}^{i}-X_{n, m}^{i}\right) \\
+c_{2} \cdot r_{2}\left(p_{\text {best }}^{i}-X_{n, m}^{i}\right)
\end{array} \\
X_{n, m}^{(i+1)}=X_{n, m}^{i}+V_{n, m}^{i}
\end{gathered}
$$

Where: $V_{n, m}$ is the velocity of particle $n$ and dimension $m ; X_{n, m}$ is the position of particle $n$ and dimension $m ; c_{1}$ and $c_{2}$ are acceleration constants; $w$ is inertia weight factor; $r_{1}$ and $r_{2}$ are random numbers; $p_{\text {best }}$ is best position of a specific particle and $g_{\text {best }}$ is best particle of the group.

The random direction in some intelligent algorithms leads to a delay in reaching the global solution. This problem can be solved by using the DPSO algorithm to speed up the optimal solution.

In this section, PSO performance can be developed by updating the new weight (Wnew) based on increasing the linearly. The proposed Wnew is evaluated as follows:

$$
\begin{aligned}
W_{\text {new }}=W_{\text {initial }} & +\left[\left(W_{\text {final }}\right.\right. \\
& \left.\left.-W_{\text {initial }}\right)\left(1-\left(\frac{h}{H}\right)^{2}\right)^{2}\right]
\end{aligned}
$$

Where: $W_{\text {initial }}$ is initial inertia weight; $W_{\text {finial }}$ is final inertia weight; $H$ is the number of iterations; and $h=1,2,3, \ldots, H$.

\section{The proposed models}

In this section, three models are proposed for wind energy output prediction, as follows:

\subsection{The proposed FNN model}

This section has suggested that a Fuzzy logic be combined with a Neural Network to create an FNN model to obtain an ideal solution to the WEOP problem. The proposed neural network model in this paper consists of two inputs and two outlets on the chassis, as depicted in Fig. 1 [6, 29].

The first layer represents the input layer, and each node corresponds to the input fixed, and the network has five layers. The input signal is sent to the next layer without conversion, which is the horizontal input layer, and is considered the second layer, and its role is to obliterate the scene change. As for the third layer, the neural network hidden layer and its primary role are the same as the regular neural network role itself used to set the fuzzy value of the input mutable to the fuzzy value of the input mutable.

Finally, the fourth layer, an output layer, ultimately represents the proposed model for wind energy output prediction. The fifth layer statistical value is a weighted output layer, which outputs a specific value. For D-dimensional inputs $e=\left(e_{1}, e_{2} \ldots\right.$ $\left.e_{m}\right)$ and used membership function of Gaussian function as follows:

$$
M A_{j}^{t}=\exp \left[-\left(e_{j}-C_{j}^{t}\right)\left(2 / \sigma_{j}\right)\right]
$$

The multiplication operators of Fuzzy membership calculations as shown in the following equation:

$$
S^{t}=M A_{j}^{1}\left(e_{1}\right) \cdot M A_{j}^{2}\left(e_{2}\right) \ldots M A_{j}^{m}\left(e_{m}\right)
$$

And the output value of the fuzzy model:

$$
\begin{aligned}
y_{t}=\sum_{t=1}^{m} S^{t}\left(P_{o}^{t}\right. & +P_{o}^{t} e_{i}+\cdots \\
& \left.+P_{k}^{t} e_{k}\right) / \sum_{t=1}^{m} S^{t}
\end{aligned}
$$

The network error equation:

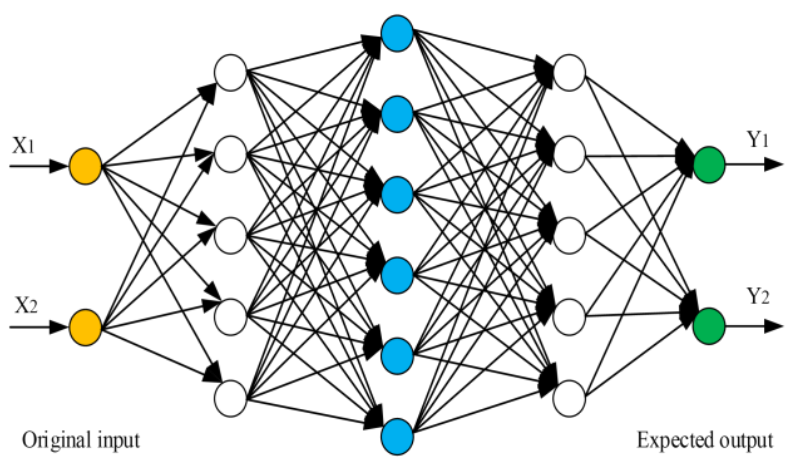

Figure. 1 Flowchart of the NN Model 


$$
E=0.5 \sum_{t=1}^{m}\left(y_{t}-L\right)^{2}
$$

Where: $c$ is center value of the Gaussian membership function; $\sigma$ is width value of the Gaussian membership function; $j=1,2, \ldots, n ; P_{o}$ is output value of fuzzy model; $t=1,2, \ldots, m ; y_{t}$ is actual output of the network; $L$ is actual output of the network, and $k$ is number of sample data.

The ANNs have benefits for supplier selection; they consider the quantitative factors instead of the qualitative factors. The reason is the difficulty of formalizing and collection. Therefore, the proposed FNN leads to a more precise decision where FNN is based on evolutionary approaches to avoid FNN getting the local minimum.

The main implementation steps of the WEOP model based on FNN are as follows:

Step1: Set which factor is the most effect on supplier selection.

Step2: Round up the quantitative and qualitative information.

Step3: Base development of the Fuzzy IF-THEN rule. Step4: Train the FNN to build a database of the fuzzy rule.

Step5: Train the integrated NN model.

\subsection{The proposed DPSO-FNN model}

The FNN Model contains a center $(c)$ value and $(\sigma)$ width of the Gaussian membership function. The value and the connection weight of the network need to be improved for training, and the error requirements are achieved by fast and suitable methods.

Moreover, the mathematics parameter correction is used for assessment and training. Still, when the data size is large the problem becomes complex and takes very long to calculate and does not give accuracy in calculating requirements. Hence, it is not possible to obtain distinct training results.

Therefore, DPSO optimization in section (3), can be used to improve the parameters of the FNN, as DPSO works to improve the performance of the FNN and make it effective. Besides, it can quickly reduce learning and generalization errors. FNN equations in section (4.1) can be rewritten after merging the DPSO model to improve its work by using the new DPSOFNN Model. Assume that in a D-dimensional input for $m$-th particles are represented as a vector:

$$
e_{t}=\left(e_{t 1}, e_{t 2}, \ldots, e_{t m}\right)
$$
follows:

Can be represented velocity of $m$-th partial as

$$
V_{t}=\left(V_{t 1}, V_{t 2}, \ldots, V_{t m}\right)
$$

The optimal position currently searched by the $m$ th particle is called the locale best value $\left(P_{\text {best }}\right)$ and written as:

$$
P_{b e s t, t}=\left(P_{t 1}, P_{t 2}, \ldots, P_{t m}\right)
$$

The optimal position currently searched by the $m$ th particle is called the global best value $\left(g_{\text {best }}\right)$ and written as:

$$
g_{\text {best }, t}=\left(g_{t 1}, g_{t 2}, \ldots, g_{t m}\right)
$$

Where: $t=1,2 \ldots m$.

The implementation steps of the DPSO-FNN Model are as follows:

Step1: Initialize the original data, and establish an appropriate network structure based on the input fuzzy.

Step2: Update the individual extreme values and extreme global values of the DPSO. The training samples are input into the FNN model. The DPSO method is used to train $c_{i t}, \sigma_{i}$ of the network. When the fitness function value reaches the error requirement, the calculation is stopped, and the FNN model is optimal at this time.

Step3: Update DPSO individual extreme and global extreme. The mean square error output from the training model is used as the fitness value of the DPSO to find the optimal value of each particle. At the same time, it is compared with the current particle optimal value $P_{i}$, and the individual optimal value of the particle is updated. If the optimal value of a single particle is better than the optimal value $p_{\text {base }}$ of all current particles, the optimal value of the current particle is used as the global optimal value $g_{\text {base }}$.

Step4: Update particle position and continuity. Update particle position and velocity in DPSO, according to Eqs. (1) and (2).

Step5: Train and test; if the training error requirement is met, terminate the selection process, then the obtained parameter is the optimal value of FNN training: if the training error requirement cannot be met, go to step-2 and iterate again. 


\subsection{The proposed DPSO-BPNN model}

The DPSO-BPNN model combines the advantages of local optimization of the BP neural network and global optimization of the PSO algorithm. The DPSO algorithm is used to optimize the weights and thresholds value of the BP network and then continuously updates the particle position and velocity until the search is optimized adapted values to improve network training speed and prediction accuracy. The steps of the proposed DPSO-BPNN model can be described as follows:

Step1: Determine the structure topology of the BPNN, that is, the number of neurons $l, n$, and $m$ in the input layer, hidden layer, and output layer.

Step2: Initialize the BPNN (weights, thresholds) and particle swarm (particle position, velocity, etc.).

$$
\begin{aligned}
& V_{i j}=V_{i j}+\mu X_{j}\left(1-X_{i}\right) p(i) \sum_{t=1}^{m} V_{j t} e_{t} \\
& V_{i t}=V_{i t}+\mu X_{j} e_{t}
\end{aligned}
$$

Where: $i=1,2, \ldots, l ; j=1,2, \ldots, n ; t=1,2, \ldots, m ; V_{i j}$ and $V_{j t}$ are the connection weights of the BP neural network; $P_{i}, X_{j}$ is the layer input and the hidden layer output, respectively; $\mu$ is the learning rate.

$$
\begin{aligned}
& b_{t}=b_{t}+e_{t} \\
& a_{j}=a_{j}+\mu X_{j}\left(1-X_{j}\right) \sum_{t=1}^{m} V_{j t} e_{t}
\end{aligned}
$$

Where: $a_{j}$ and $b_{t}$ national values of hidden layer and output layer

Step3: Calculate the fitness value $(F)$ of the particles.

$$
F=\frac{1}{S} \sum_{i=1}^{l} \sum_{j=1}^{N}\left(w_{j i}^{1}-w_{j i}^{2}\right)^{2}
$$

Where: $S$ is the number of training samples; $N$ is the number of neurons in the output network; $w p_{j i}^{1}$ is the ideal wind power output value of the $j$-th network output node of the $i$ sample; $w p_{j i}^{2}$ is the actual wind power output value of the $j$-th network output node of the $i$ sample.

Step4: Search for individual extreme values $p_{\text {best }}$ of the particles and global extreme values $g_{\text {best }}$. If the current fitness value is better than $g_{\text {best }}$, update the particles $g_{\text {best }}$, if not, update the velocity and position of the particles.

If the current fitness value of all particles is better than $p_{\text {best }}$, then update $p_{\text {best }}$.

Step5: Update the velocity and position of the particles according to Eqs. (1) and (2).

Step6: Update the weight Wnew according to Eq. (3). Step7: Check if the termination conditions are met. If it is, stop iteration to obtain the optimal weights and thresholds of the BPNN. Otherwise, return to (step3) and recalculate the fitness value of the particles.

Step8: Train and test the BPNN; calculate the error, update the weights and thresholds values, and check if the end condition is met (whether the current position or number of iterations reaches a predetermined error).

If it is, stop iteration and output the neural network optimal weights and thresholds; otherwise, recalculate the error.

Fig. 2 shows a flowchart of the proposed DPSOBPNN model.

\section{Simulation results and discussion}

\subsection{Data processing and criteria of prediction accuracy}

In this paper, the information contained in the reference $[17,18]$ for two cases (48 hours and 72 hours) wind energy data resolution was relied upon for study and comparison. Therefore, it is suitable for the performance testing and feasibility of the proposed prediction models. It can also be noted that the proposed models adopted in this paper have been implemented using MATLAB/2020a software.

This paper selects three accuracy measures to evaluate the effectiveness and practicability of the proposed prediction model performance. The performance measures of RMSE, MAPE, and MAE are utilized to quantify the errors of predicted values, and the smaller, the better the prediction capability that the model has.

The computational formulas of these three criteria of prediction accuracy measure are formulated as follows [34]:

$$
\begin{aligned}
& \text { RMSE }=\sqrt{\frac{1}{T} \sum_{t=1}^{T}\left(p_{t}-\hat{p}_{t}\right)^{2}} \\
& M A P E=\frac{1}{T} \int_{t=1}^{T}\left|\frac{p_{t}-\hat{p}_{t}}{p_{t}}\right|
\end{aligned}
$$


$M A E=\frac{1}{T} \int_{t=1}^{T}\left|p_{t}-\hat{p}_{t}\right|$

Where: $T$ is the total of test data, $p_{t}$ is the real value of the wind power at the time $(t)$, and $\hat{p}_{t}$ is predicted value of the wind energy at time $(t)$.

\subsection{Model Validate}

In this part, the superiority of the proposed models in predicted capability can be verified by adopting the FNN, DPSO-FNN, and DPSO-BPNN

Three error measurements, RMSE, MAPE, and $M A E$, are employed for performance accuracy evaluating of all the predicting models. Fig. 3, 4 describes the predicted results of the proposed models. Moreover, Table 1 illustrates the predicted errors, including RMSE, MAPE, and MAE of the proposed models. The predicted error values of $M A E$, $R M S E$, and MAPE of the proposed DPSO-BPNN model

illustrated in Table 1 and Table 2 are small compared with all the proposed models in this paper and proposed models in reference $[17,18]$.

It confirmed that the proposed DPSO-BPNN model had the best predicting performance.

For more comparison, Fig. 5 and Fig. 6 show the errors of RMSE, MAPE, and MAE of various models for two cases (48 Hours and 72 Hours).

According to the results illustrated in Table 1, Table 2, Fig. 5, and Fig.6, four comparisons of results can be obtained as follows:

(a) When comparing DPSO-BPNN vs. FNN and DPSO-BPNN vs. DPSO-FNN as in Table 1 and Fig. 5. We will note MAE, RMSE, and MAPE values have improved for the DPSO-BPNN model, about $96.79 \%, 94.51 \%$, and $73.54 \%$ compared to the FNN model, and $91.89 \%, 92.07 \%$, and $62.96 \%$ compared to the DPSO-FNN model.

Therefore, based on the above analysis, it can be easily found that the proposed DPSO-BPNN model can significantly decrease the errors, including MAE, RMSE, and MAPE of FNN and DPSO-FNN models. Thus, it can be concluded that the proposed DPSOBPNN is very effective for performance, improving the criteria of prediction accuracy.

(b) To make sure that the development of the PSO algorithm on the BPNN model proves its effectiveness compared to DPSO-BPNN vs. IDASVM. Where the IDA-SVM has considered the best model suggested in the reference [30]. We'll note from Table 1 and Fig. 5 that the values of MAE, RMSE, and MAPE of the DPSO-BPNN model have been improved about $90.68 \%, 85.78 \%$, and $63.68 \%$ compared to the IDA-SVM model [17], and it is a good indication of the success of the method proposed in this paper.

(c) According to the results illustrated in Table 2 and Fig. 6. In comparison the DPSO-BPNN vs. FNN and DPSO-BPNN vs. DPSO-FNN, it can be found that the values of MAE, RMSE, and MAPE of the DPSO-BPNN model improved about $98.51 \%$, $98.19 \%$, and $89.63 \%$ compared to the FNN model, and approximately $94.69 \%, 95.89 \%$, and $83.14 \%$ compared to the DPSO-FNN model.

Based on the above comparison, it can be concluded that the integrating of the DPSO algorithm into the FNN and BPNN models can decrease the nonlinearity and non-stability characteristics that are occurred in the original wind energy series to some extent. Therefore, it is useful and suitable for improving the predicting ability of FNN and BPNN models.

(d) When compare DPSO-BPNN vs. ARIMANN. Where the ARIMA-NN has considered the best model suggested in the reference [18]. We'll note from Table 2 and Fig. 6 that RMSE and MAPE values by approximately $94.04 \%$ and $14.98 \%$, respectively, compared with the ARIMA-NN model [18]. We can be noted that through optimizing the neural network, the initial weights and thresholds are optimized by the DPSO algorithm, enabling the BPNN model to obtain

Table 1. Comparison of prediction performances among various models (48 Hours)

\begin{tabular}{|l|c|c|c|}
\hline Models Index & MAE & $\boldsymbol{R M S E}$ & $\boldsymbol{M A P E}$ \\
\hline Proposed FNN & 7.97 & 8.42 & 14.52 \\
\hline Proposed DPSO-FNN & 3.16 & 5.83 & 10.37 \\
\hline Proposed DPSO-BPNN & $\mathbf{0 . 2 5 6 2}$ & $\mathbf{0 . 4 6 2 1}$ & $\mathbf{3 . 8 4 1 6}$ \\
\hline Grid-SVM [17] & 3.09 & 3.80 & 10.84 \\
\hline GA-SVM [17] & 3.48 & 4.20 & 12.54 \\
\hline DA-SVM [17] & 3.68 & 4.42 & 13.06 \\
\hline IDA-SVM [17] & 2.75 & 3.25 & 10.58 \\
\hline
\end{tabular}

Table 2. Comparison of prediction performances among various models (72 Hours)

\begin{tabular}{|l|c|c|c|}
\hline \multicolumn{1}{|c|}{ Index } & MAE & $\boldsymbol{R M S E}$ & $\boldsymbol{M A P E}$ \\
\hline Proposed FNN & 8.65 & 9.79 & 27.88 \\
\hline Proposed DPSO-FNN & 2.42 & 4.31 & 11.93 \\
\hline Proposed DPSO-BPNN & $\mathbf{0 . 1 2 8 5}$ & $\mathbf{0 . 1 7 7 2}$ & $\mathbf{2 . 0 1 1}$ \\
\hline RBPNN [18] & --- & 18.729 & 16.736 \\
\hline ARIMA [18] & --- & 28.743 & 27.183 \\
\hline BPNN [18] & --- & 23.877 & 22.032 \\
\hline ARIMA-NN [18] & --- & 3.133 & 2.659 \\
\hline
\end{tabular}




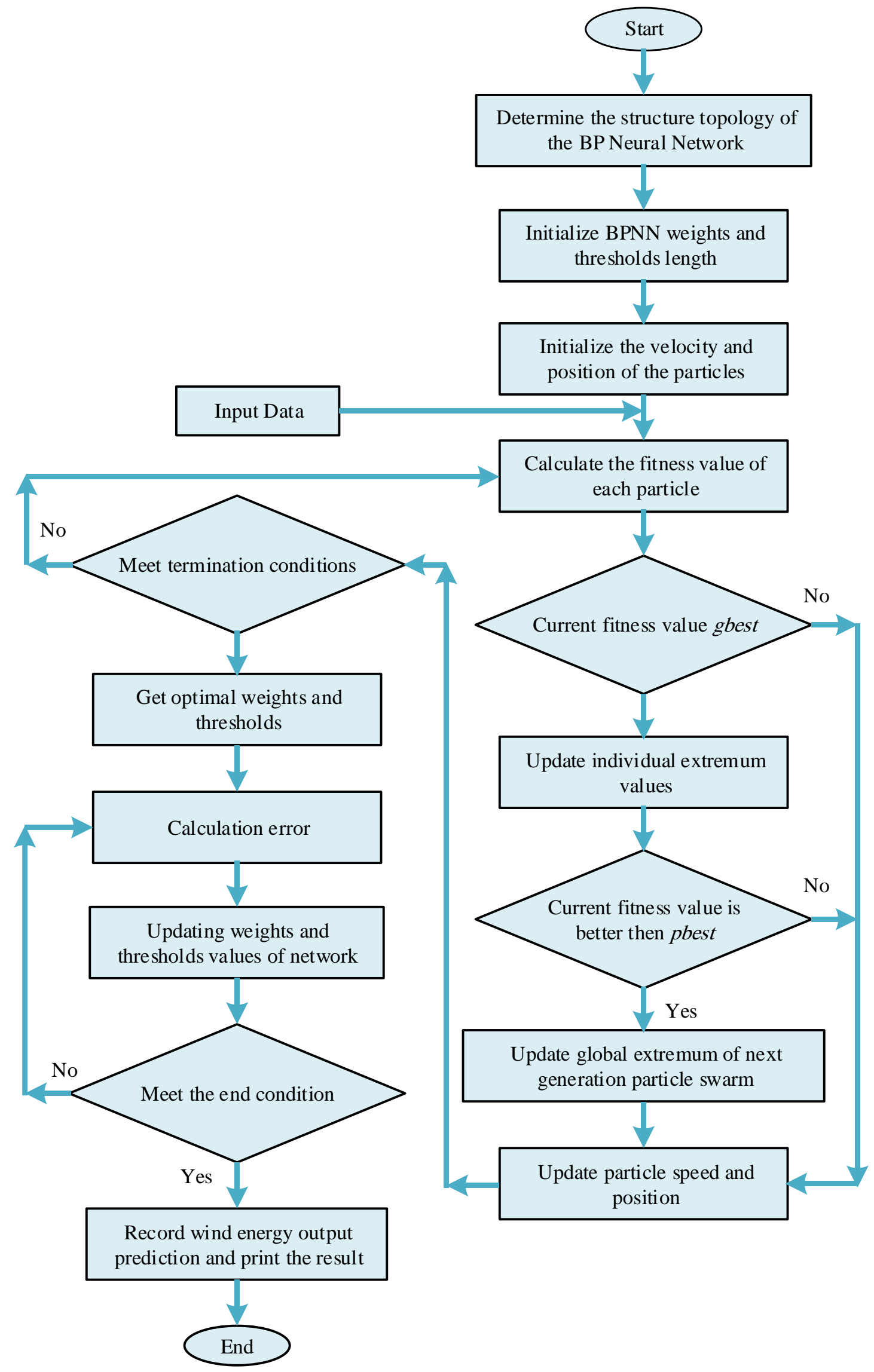

Figure. 2 Flowchart of DPSO-BPNN mode 


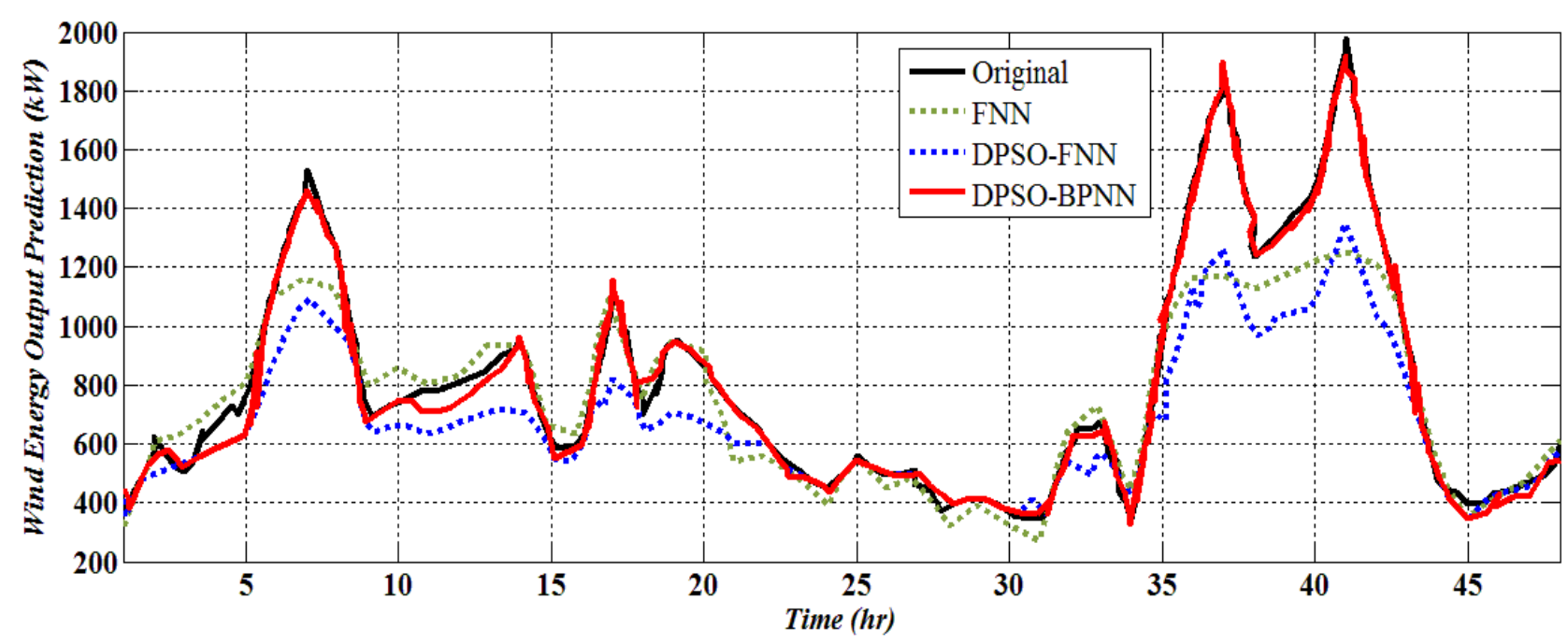

Figure. 3 The definitive predict result of various proposed models (48 Hours)

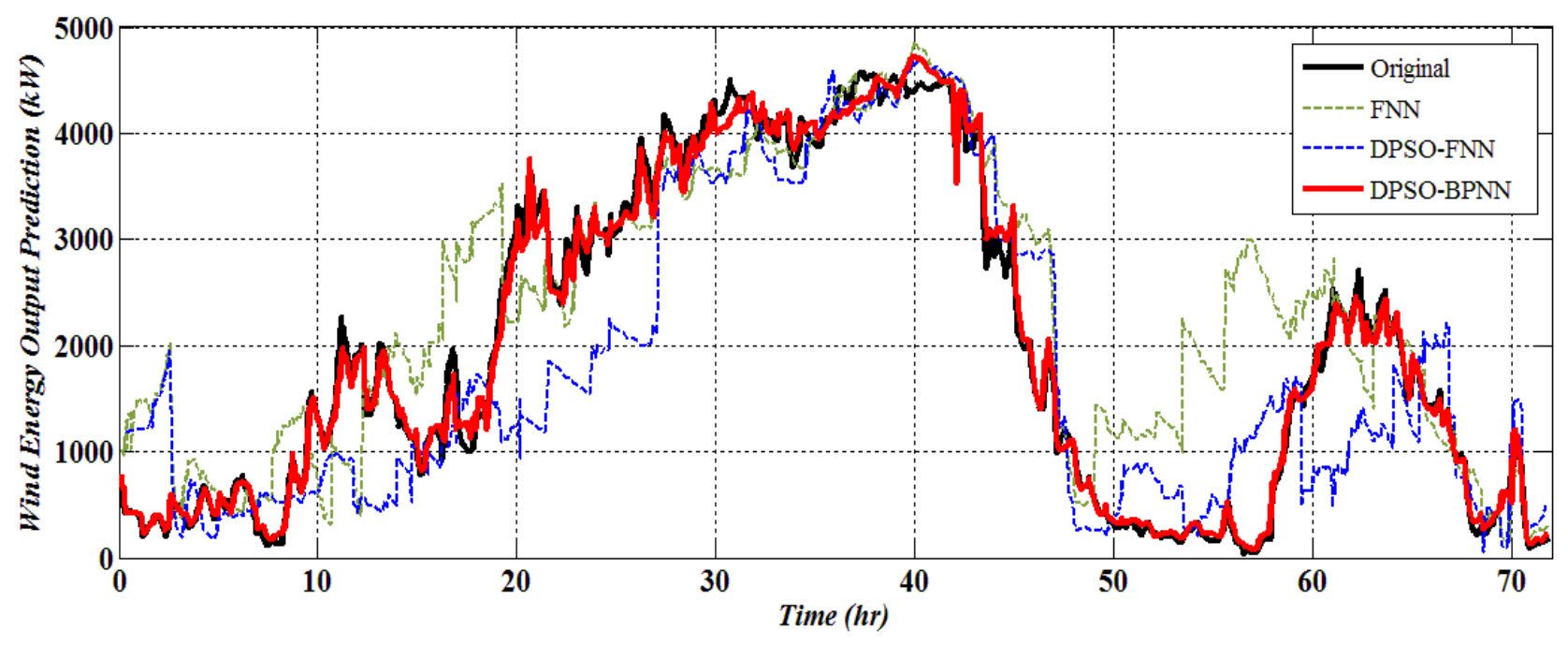

Figure. 4 The definitive predict result of various proposed models (72 Hours)

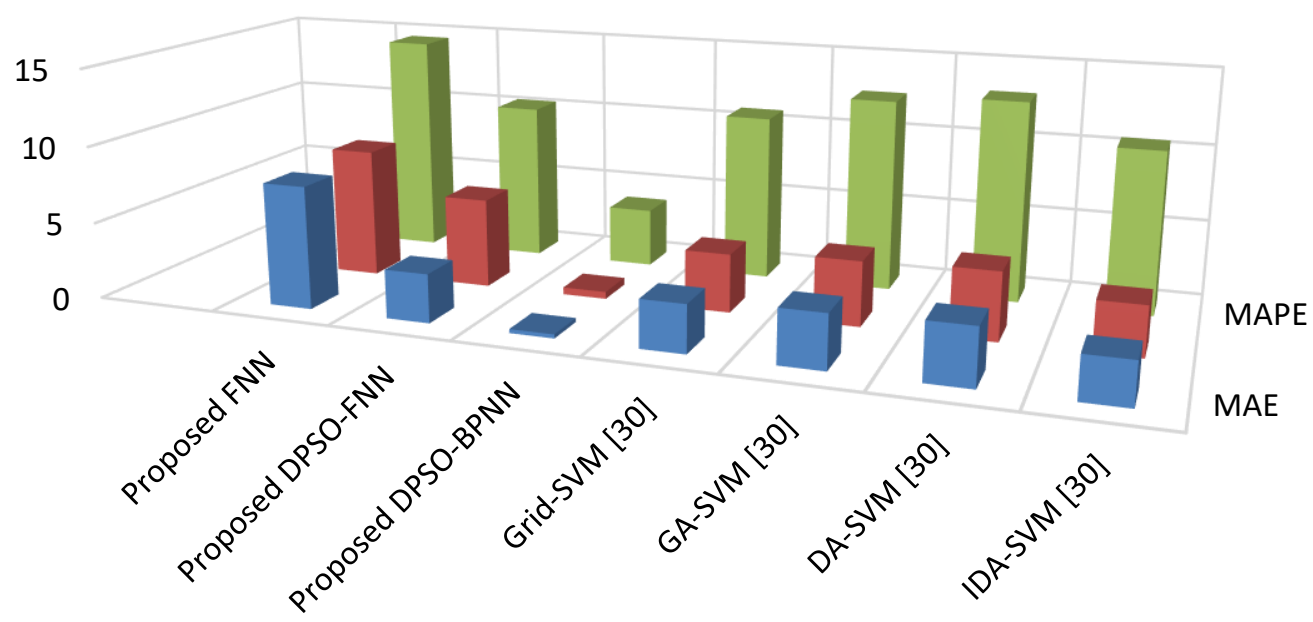

Figure. 5 Error graphics of various models (48 Hours) 


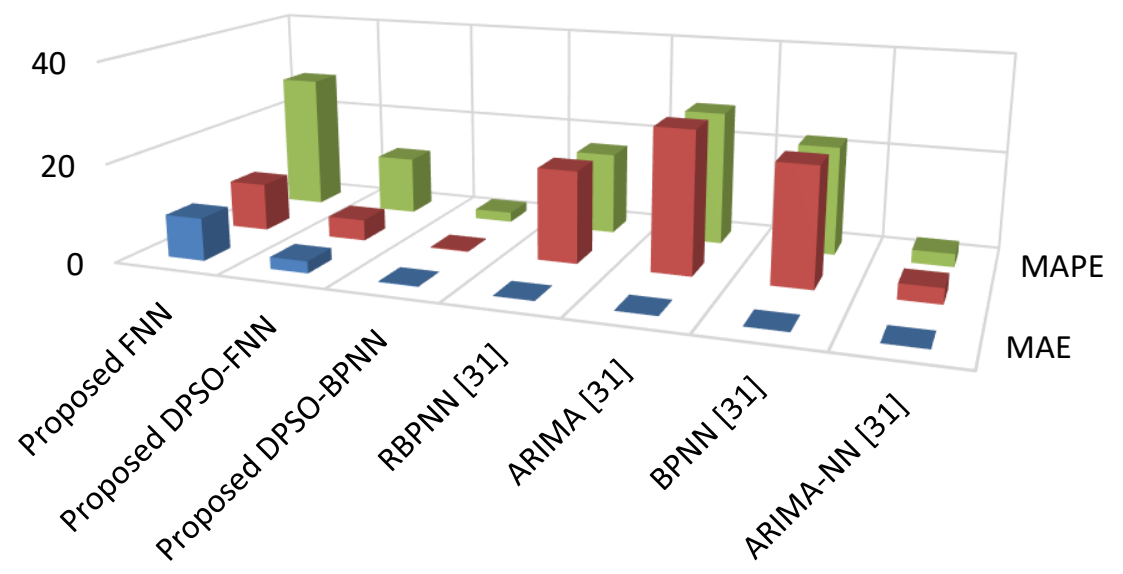

Figure. 6 Error graphics of various models (72 Hours)

a stronger approximation. Therefore, it is found that the Wind energy output predicting model based on the DPSO-BPNN model used in this paper shows a positive effeteness and better prediction accuracy.

\section{Conclusions}

This paper proposed three models to increase the WEOP. The suggested prediction models consist of the FNN model, DPSO-FNN model, and DPSOBPNN model. The simulation results show that the proposed DPSO-BPNN model has higher prediction accuracy than the FNN model and DPSO-FNN model and is suitable for short-term WEOP. Therefore, the results of this paper are as follow:

1) Development of PSO algorithm was used by modifying the initial weights (Wnew) and adopting the BPNN algorithm. The hybrid DPSO-BPNN model is evaluated to predict the WEOP in the short-term.

2) The proposed models were trained by actual measured data to predict the wind energy output in terms of the short-term.

3) The proposed DPSO-BPNN model can effectively improve wind power prediction accuracy compared with the FNN and DPSO-FNN models.

4) The proposed DPSO-BPNN model was compared with the models presented in previous literature, such as Grid-SVM, GA-SVM, DA-SVM, IDA-SVM [17], RBPNN, ARIMA, BPNN, and ARIMA-NN [18], to emphasize the proposed model's effectiveness and applicability.

(5) This paper selects three accuracy measures to evaluate the performance, effectiveness and practicability of the proposed model. The three prediction errors and accuracy of the DPSO-BPNN model are superior compared with the proposed models in this paper and presented in references [17, 18]. The prediction error rate was improved by about $98 \%$.
Two essential conclusions are obtained as follows:

Firstly, the proposed DPSO-BPNN model has the best performance compared with other considered benchmark models. The proposed model shows have training speed and high prediction accuracy, providing technical support for future dispatching operations and ensuring the power system stable and safe operation.

Secondly, the proposed DPSO algorithm shows a positive effect on the BPNN model by optimizing the initial weights and thresholds between the input and hidden layers.

For future work, there are many research directions. For example, plan further study for longer-term wind energy output prediction. Some intelligent optimization methods can be combined and meteorological factors to improve prediction accuracy. Finally, we can be applying the proposed model to other fields, such as predict electricity consumers' loads and wind speed output.

\section{Conflicts of Interest}

The authors confirm that there is no conflict of interest. approximately

\section{Author Contributions}

The following statements should be used as follows: "Conceptualization, Mohammed Kdair Abd and Dhari Y. Mahmood; methodology, Mohammed Kdair Abd; software, Mohammed Kdair Abd; validation, Mohammed Kdair Abd, Dhari Y. Mahmood, and Kanaan A. Jalal; formal analysis, Mohammed Kdair Abd; investigation, Kanaan A. Jalal; resources, Dhari Y. Mahmood; data curation, Mohammed Kdair Abd; writing — original draft preparation, Mohammed Kdair Abd; writingreview and editing, Mohammed Kdair Abd, Dhari Y. 
Mahmood, and Kanaan A. Jalal; visualization, Kanaan A. Jalal; supervision, Dhari Y. Mahmood; project administration, Mohammed Kdair Abd".

\section{References}

[1] P. A. Adedeji, S. Akinlabi, N. Madushele, and O. O. Olatunji, "Wind turbine power output very short-term forecast: A comparative study of data clustering techniques in a PSO-ANFIS model", Journal of Cleaner Production, Vol. 254, 2020.

[2] Z. Sun, H. Sun, and J. Zhang, "Multistep wind speed and wind power prediction based on a predictive deep belief network and an optimized random forest", Mathematical Problems in Engineering, 2018.

[3] F. Cassola, and M. Burlando, "Wind speed and wind energy forecast through Kalman filtering of Numerical Weather Prediction model output", Applied energy, Vol. 99, pp. 154-166, 2012.

[4] Y. Tian, Q. Liu, Z. Hu, and Y. Liao, "Wind speed forecasting based on Time seriesAdaptive Kalman filtering algorithm", In: IEEE Far East Forum on Nondestructive Evaluation/Testing, pp. 315-319, 2014.

[5] Y. Zhang, H. xu Sun, Y. jun Guo, and Z. ping $\mathrm{Lu}$, "Improved grey model with rolling method for wind power prediction", In: 35th Chinese Control Conference (CCC), pp. 9784-9786, 2016.

[6] G. Osório, J. Matias, and J. Catalão, "Short-term wind power forecasting using adaptive neurofuzzy inference system combined with evolutionary particle swarm optimization, wavelet transform and mutual information", Renewable Energy, Vol. 75, pp. 301-307, 2015.

[7] Wang, J. Hu, K. Ma, and Y. Zhang, "A selfadaptive hybrid approach for wind speed forecasting", Renewable Energy, Vol. 78, pp. 374-385, 2015.

[8] G.-Q. Lin, L.-L. Li, M.-L. Tseng, H.-M. Liu, D.D. Yuan, and R. R. Tan, "An improved mothflame optimization algorithm for support vector machine prediction of photovoltaic power generation", Journal of Cleaner Production, Vol. 253, 2020.

[9] H. Wang, W. Xue, Y. Liu, J. Peng, and H. Jiang, "Probabilistic wind power forecasting based on spiking neural network", Energy, Vol. 196, 2020.

[10] H. Demolli, A. S. Dokuz, A. Ecemis, and M. Gokcek, "Wind power forecasting based on daily wind speed data using machine learning algorithms", Energy Conversion and Management, Vol. 198, 2019.
[11] H. D. Kabir, M. A. Hosen, A. Khosravi, and S. Nahavandi, "Weighted Autocorrelation based Prediction Interval Optimization for Wind Power Generation", In: International Joint Conference on Neural Networks (IJCNN), pp. 16, 2018.

[12] Z. Yang, and J. Wang, "A combination forecasting approach applied in multistep wind speed forecasting based on a data processing strategy and an optimized artificial intelligence algorithm", Applied Energy, Vol. 230, pp. 1108$1125,2018$.

[13] G. Grassi and P. Vecchio, "Wind energy prediction using a two-hidden layer neural network", Communications in Nonlinear Science and Numerical Simulation, Vol. 15, pp. 2262-2266, 2010.

[14] P. Lu, L. Ye, W. Zhong, Y. Qu, B. Zhai, Y. Tang, et al., "A novel spatio-temporal wind power forecasting framework based on multi-output support vector machine and optimization strategy", Journal of Cleaner Production, Vol. 254, 2020.

[15] S. Wang, J. Yang, F. Li, and T.-1. LIU, "Shortterm wind speed forecasting based on EMD and ANN", Power System Protection and Control, Vol. 40, pp. 6-11, 2012.

[16] P. Lu, L. Ye, B. Sun, C. Zhang, Y. Zhao, and J. Teng, "A new hybrid prediction method of ultrashort-term wind power forecasting based on EEMD-PE and LSSVM optimized by the GSA. Energies, Vol. 11, No. 4, pp. 697, 2018.

[17] Li, L.L., Zhao, X., Tseng, M.L., and Tan, R.R., "Short-term wind power forecasting based on support vector machine with improved dragonfly algorithm", Journal of Cleaner Production, Vol. 242, 2020.

[18] G. Chang, H. Lu, L. Hsu, and Y. Chen, "A hybrid model for forecasting wind speed and wind power generation", In: IEEE Power and Energy Society General Meeting (PESGM), pp. 1-5, 2016.

[19] Zhao, S. Wang, and T. Li, "Review of evaluation criteria and main methods of wind power forecasting", Energy Procedia, Vol. 12, pp. 761769, 2011.

[20] M. G. Lobo, and I. Sanchez, "Aggregated wind power prediction methods based on distances between weather forecasting vectors", In: International Conference on Clean Electrical Power, pp. 242-247, 2009.

[21] X. Wang, P. Guo, and X. Huang, "A review of wind power forecasting models", Energy procedia, Vol. 12, pp. 770-778, 2011. 
[22] Guan, J., Lin, J., Guan, J., and Mokaramian, E., "A novel probabilistic short-term wind energy forecasting model based on an improved kernel density estimation", International Journal of Hydrogen Energy, Vol. 45, No. 43, pp. 2379123808, 2020.

[23] S. Salcedo-Sanz, A. M. Perez-Bellido, E. G. Ortiz-García, A. Portilla-Figueras, L. Prieto, and D. Paredes, "Hybridizing the fifth generation mesoscale model with artificial neural networks for short-term wind speed prediction", Renewable Energy, Vol. 34, pp. 1451-1457, 2009.

[24] Li, C., Tang, G., Xue, X., Chen, X., Wang, R. and Zhang, C., "The short-term interval prediction of wind power using the deep learning model with gradient descend optimization", Renewable Energy, Vol. 155, pp. 197-211, 2020.

[25] N. Shabbir, R. AhmadiAhangar, L. Kütt, M. N. Iqbal, and A. Rosin, "Forecasting Short Term Wind Energy Generation using Machine Learning", In: IEEE 60th International Scientific Conference on Power and Electrical Engineering of Riga Technical University (RTUCON), pp. 1-4, 2019.

[26] S. Xiao, J. Lu, D. Li, H. Yu, M. Li, and J. Liu, "Long-term wind power forecasting model by multi meteorological variables based on data compensation", In: 2nd International Conference on Power and Renewable Energy (ICPRE), pp. 451-455, 2017.

[27] T. G. Barbounis, J. B. Theocharis, M. C. Alexiadis, and P. S. Dokopoulos, "Long-term wind speed and power forecasting using local recurrent neural network models", IEEE Transactions on Energy Conversion, Vol. 21, pp. 273-284, 2006.

[28] T. Barbounis, and J. B. Theocharis, "Locally recurrent neural networks for long-term wind speed and power prediction", Neurocomputing, Vol. 69, pp. 466-496, 2006.

[29] B. Zhu, M.-y. Chen, N. Wade, and L. Ran, "A prediction model for wind farm power generation based on fuzzy modeling", Procedia Environmental Sciences, Vol. 12, pp. 122-129, 2012.

[30] A. Clifton, L. Kilcher, J. K. Lundquist, and P. Fleming, "Using machine learning to predict wind turbine power output", Environmental research letters, Vol. 8, 2013.

[31] E. Vladislavleva, T. Friedrich, F. Neumann, and M. Wagner, "Predicting the energy output of wind farms based on weather data: Important variables and their correlation", Renewable energy, Vol. 50, pp. 236-243, 2013.

[32] A. Kollu, and S. Vadlamudi, "Eagle Strategy with Cauchy Mutation Particle Swarm Optimization for Energy Management in Cloud Computing", International Journal of Intelligent Engineering and Systems, Vol. 13, No. 6, 2020.

[33] R. H. Al-Rubayi, M. K. Abd, and F. M. Flaih, "A New Enhancement on PSO Algorithm for Combined Economic-Emission Load Dispatch Issues", International Journal of Intelligent Engineering and Systems, Vol. 13, No. 2, pp. 7785, 2020.

[34] D. Wang, Y. Liu, H. Luo, C. Yue, and S. Cheng, "Day-ahead PM2. 5 concentration forecasting using WT-VMD based decomposition method and back propagation neural network improved by differential evolution", International journal of environmental research and public health, Vol. 14, p. 764, 2017. 
Notation list

\begin{tabular}{|c|c|}
\hline Symbol & Parameters \\
\hline$a_{j}, b_{t}$ & $\begin{array}{l}\text { National values of hidden layer and } \\
\text { output layer }\end{array}$ \\
\hline$c$ & $\begin{array}{l}\text { Center of the Gaussian membership } \\
\text { function. }\end{array}$ \\
\hline$c_{1}, c_{2}$ & Acceleration constants \\
\hline$e$ & D-dimensional inputs \\
\hline$E$ & Network error \\
\hline$\overline{e_{t}}$ & D-dimensional input for $m$-th particles \\
\hline$F$ & Fitness value of the particles. \\
\hline$g_{\text {best }}$ & Particle of the group \\
\hline$H$ & Number of iterations \\
\hline$k$ & Number of sample data \\
\hline$L$ & Actual output of the network \\
\hline$N$ & $\begin{array}{l}\text { Number of neurons in the output } \\
\text { network }\end{array}$ \\
\hline$p_{\text {best }}$ & Best position of a specific particle \\
\hline$P_{i}, X_{j}$ & Layer input and hidden layer output \\
\hline$P_{o}$ & Output value of fuzzy model \\
\hline$\hat{p}_{t}$ & $\begin{array}{l}\text { Predicted value of wind power at time } \\
(t)\end{array}$ \\
\hline$p_{t}$ & Real value of wind power at time $(t)$ \\
\hline$r_{1}, r_{2}$ & Random numbers \\
\hline$S$ & Number of training samples \\
\hline$S^{t}$ & $\begin{array}{l}\text { Multiplication operators of Fuzzy } \\
\text { membership }\end{array}$ \\
\hline$T$ & Total of test data \\
\hline$V_{i j}, V_{j t}$ & $\begin{array}{l}\text { Connection weights of the BP neural } \\
\text { network }\end{array}$ \\
\hline$V_{n, m}$ & Velocity of particle $n$ and dimension $m$ \\
\hline $\mathrm{W}$ & Inertia weight factor \\
\hline$W_{\text {finial }}$ & Final inertia weight \\
\hline$W_{\text {initial }}$ & Initial inertia weight \\
\hline$w p_{j i}^{1}$ & $\begin{array}{l}\text { Ideal wind power output value of the } j \text { - } \\
t h \text { network output node of the } i \text { sample }\end{array}$ \\
\hline$w p_{j i}^{2}$ & $\begin{array}{l}\text { Actual wind power output value of the } \\
j \text {-th network output node of the } i \\
\text { sample }\end{array}$ \\
\hline$X_{n, m}$ & Position of particle $n$ and dimension $m$ \\
\hline$\mu$ & Learning rate \\
\hline$\sigma$ & $\begin{array}{l}\text { Width of the Gaussian membership } \\
\text { function. }\end{array}$ \\
\hline
\end{tabular}

